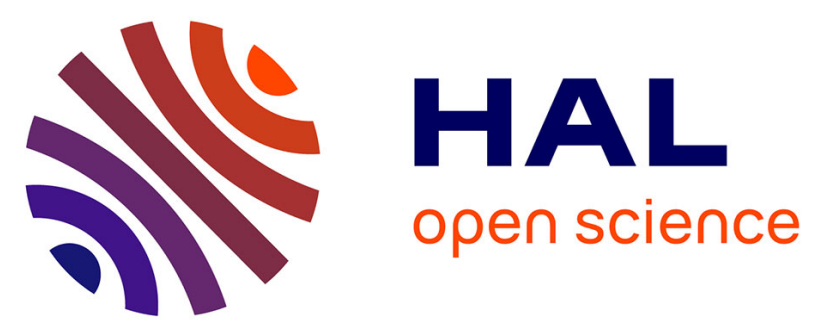

\title{
An integrated functional and transcriptomic analysis reveals that repeated exposure to diesel exhaust induces sustained mitochondrial and cardiac dysfunctions
}

\author{
Ahmed Karoui, Clément Crochemore, Paul Mulder, David Preterre, Fabrice \\ Cazier, Dorothée Dewaele, Cécile Corbière, Malik Mekki, Cathy Vendeville, \\ Vincent Richard, et al.
}

\section{To cite this version:}

Ahmed Karoui, Clément Crochemore, Paul Mulder, David Preterre, Fabrice Cazier, et al.. An integrated functional and transcriptomic analysis reveals that repeated exposure to diesel exhaust induces sustained mitochondrial and cardiac dysfunctions. Environmental Pollution, 2019, 246, pp.518-526. 10.1016/j.envpol.2018.12.049 . hal-01978723

\section{HAL Id: hal-01978723}

\section{https://hal-univ-rennes1.archives-ouvertes.fr/hal-01978723}

Submitted on 14 Jan 2019

HAL is a multi-disciplinary open access archive for the deposit and dissemination of scientific research documents, whether they are published or not. The documents may come from teaching and research institutions in France or abroad, or from public or private research centers.
L'archive ouverte pluridisciplinaire HAL, est destinée au dépôt et à la diffusion de documents scientifiques de niveau recherche, publiés ou non, émanant des établissements d'enseignement et de recherche français ou étrangers, des laboratoires publics ou privés. 


\section{An integrated functional and transcriptomic analysis reveals that repeated exposure to diesel exhaust induces sustained mitochondrial and cardiac dysfunctions}

Ahmed Karoui $^{1 \neq}$, Clément Crochemore ${ }^{1 \neq}$, Paul Mulder ${ }^{2}$, David Preterre ${ }^{3}$, Fabrice Cazier ${ }^{4}$, Dorothée Dewaele $^{4}$, Cécile Corbière ${ }^{1}$, Malik Mekki ${ }^{1}$, Cathy Vendeville ${ }^{1}$, Vincent Richard ${ }^{2}$, Jean-Marie Vaugeois ${ }^{1}$, Olivier Fardel $^{5,6}$, François Sichel ${ }^{1,7}$, Valérie Lecureur ${ }^{5}$, Christelle Monteil $^{1 *}$

1: Normandie Univ, UNIROUEN, UNICAEN ABTE, 14000 Caen et 76000 Rouen, France

2: Normandie Univ, UNIROUEN, Institut National de la Santé et de la Recherche Médicale U1096, Rouen, France.

3: CERTAM, 1 rue Joseph Fourier, 76800 Saint-Etienne du Rouvray, France

4: Common Center of Measurements (CCM), Univ. Littoral Côte d'Opale, 59140 Dunkerque, France

5: Univ Rennes, CHU Rennes, Inserm, EHESP, Irset (Institut de recherche en santé, environnement et travail) - UMR_S 1085, 35000 Rennes, France

6: Pôle Biologie, Rennes University Hospital, 35203, Rennes, France

7: Centre François Baclesse, 14000 Caen, France

$\neq$ contributed equally

*Corresponding Author

Christelle Monteil

ABTE-ToxEMAC

UFR Santé

22 Boulevard Gambetta

76000 Rouen

Phone: (00)33 235148475

Email: christelle.monteil@ univ-rouen.fr 
1

2 3

\section{Abstract}

Diesel exhaust (DE) contributes to air pollution, an important risk factor for cardiovascular diseases. However, the mechanisms by which DE exposure induces cardiovascular dysfunction remain unknown and there is still debate on the contribution of the primary particulate matter (PM) fraction compared to the gaseous phase. Although the mitochondria play a key role in the events leading to cardiovascular diseases, their role in DE-induced cardiovascular effects has not been investigated. The aim of this study was to highlight cardiac and mitochondrial events that could be disrupted following acute and/or repeated DE exposures and the contribution of gaseous pollutants to these effects. To address this question, Wistar rats were exposed to DE generated under strictly controlled and characterized conditions and extracted upstream or downstream of the diesel particulate filter (DPF). Evaluation of the cardiac function after acute DE exposure showed a disturbance in echocardiographic parameters, which persisted and worsened after repeated exposures. The presence of the DPF did not modify the cardiovascular dysfunction revealing an important implication of the gas phase in this response. Surprisingly, redox parameters were not altered by DE exposures while an alteration in mitochondrial oxidative capacity was observed. Exploration of the mitochondrial function demonstrated a more specific alteration in complex I of the respiratory chain after repeated exposures, which was further confirmed by transcriptional analysis of left ventricular (LV) tissue. In conclusion, this work provides new insights into cardiovascular effects induced by DE, demonstrating a cardiac mitochondrial impairment associated with the gaseous phase. These effects suggest deleterious consequences in terms of cardiac function for vulnerable populations with underlying energy deficit such as patients with heart failure or the elderly.

KEYWORDS: diesel exhaust, particles, cardiovascular, mitochondria, gene expression 

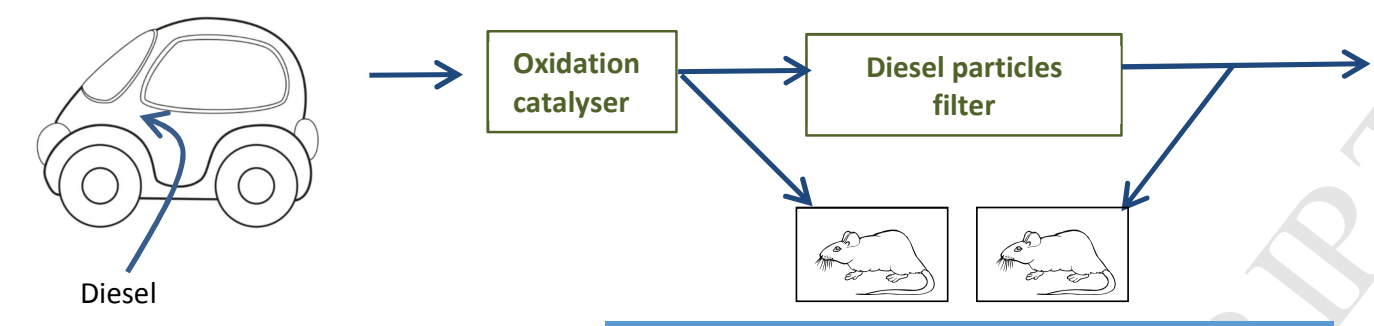

Acute (3h)

repeated

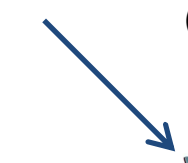

(3 weeks; 5 days/week, 3h/day)

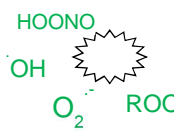

Echocardiography
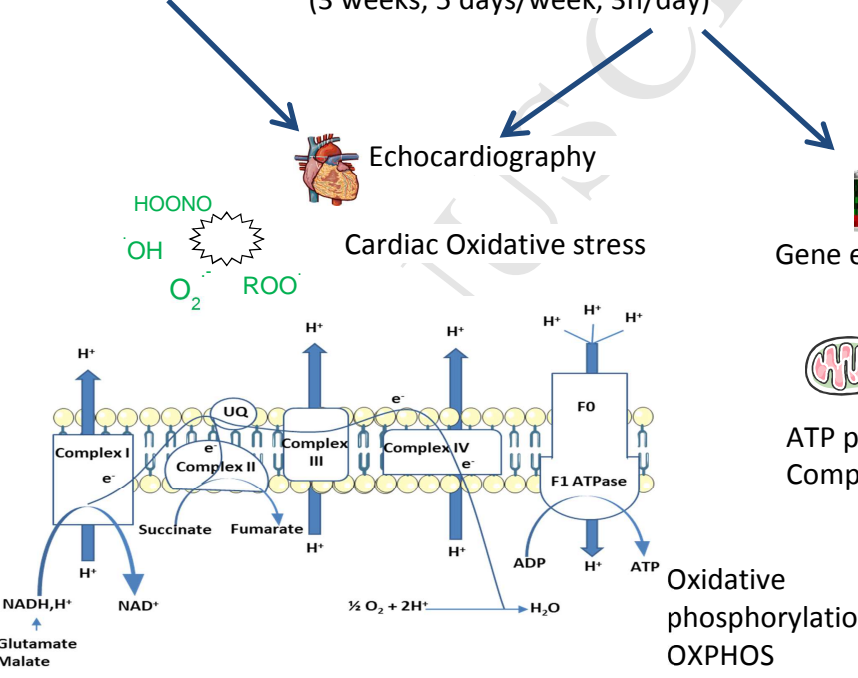

Cardiac Oxidative stress

Gene expression

CAPSULE

Diesel exhaust induces an acute cardiovascular response, which leads to a sustained cardiac mitochondrial defect and cardiac dysfunction after repeated exposures.

\section{HIGHLIGHTS}

- $\quad$ Acute and repeated diesel exhaust (DE) exposures induced a cardiac dysfunction in

rats

Repeated DE exposures induced a decrease in OXPHOS capacity 
44 - Both whole DE and gaseous phase contribute to these cardiac and mitochondrial 45 defects

46 - $\quad$ Parameters related to cardiac oxidative stress are not affected in these conditions. 
Air pollution is a major environmental risk to health and resulted in almost 9 million premature deaths in 2015 (Burnett et al., 2018). Fuel combustion contributes $85 \%$ of the particulate pollution and almost all the nitrogen oxide and sulphur (Landrigan et al., 2018). Vehicular exhaust, including diesel exhaust (DE), are responsible for a large part of the air pollution, and epidemiologic data link particulate matter (PM) levels to adverse cardiovascular effects, for both acute events (Link et al., 2013; Ye et al., 2016) and chronic exposures (Mordukhovich et al., 2015; Puett et al., 2009). It is well-documented that PM air pollution is associated with a large number of cardiovascular effects such as endothelial dysfunction (Krishnan et al., 2012; Pope et al., 2016), increased blood pressure (Honda et al., 2018; Ibald-Mulli et al., 2001), accelerated arterial thrombosis (Nemmar et al., 2003), autonomic imbalance and arrhythmias (Carll et al., 2012; Folino et al., 2017).

Given the growing concern about the health effects of particulate emissions from DE, diesel engine and fuel technology advances have been made to response to the evolution of emission regulations. These advances resulted in changes to the composition of DE with, for example, lower particulate mass (less than 1\%) with the use of diesel particulate filters (DPF) (McClellan et al., 2012). These changes in DE compositions are likely to have an impact on the cardiovascular effects of diesel engine exhaust inhalation. However, a limited number of studies have investigated the impact of these new emissions on the cardiovascular responses. The Advanced Collaborative Emissions Study (ACES) analysed new DE technology in healthy rats exposed for 24 months in order to assess plasma markers of vascular inflammation, thrombosis, cardiac fibrosis and aorta morphometry and the results showed limited effects (Conklin et al., 2015). In human, compared to untreated DE exposure, DE exposure in the presence of a DPF has been associated with a lack of acute cardiovascular effects (impaired vasodilation and ex-vivo thrombus formation) (Lucking et al., 2011). These 
results agree with the acute beneficial effects of reducing particle emissions from diesel engines. However, in another study, Karthikeyan et al. (Karthikeyan et al., 2013) demonstrated that catalyzed DPF exhaust resulted in heightened injury and inflammation due to an increase in nitrogen dioxide emissions and the release of ultrafine particles. These results outline the need for further toxicological assessments in order to understand the underlying mechanisms and identify the specific components that induce health effects.

Several biological mechanisms behind the DE-induced cardiovascular effects have previously been suggested and are generally assumed to play a key role, such as a disturbance of the systemic autonomic nervous system, translocation of PM or particles compounds into the systemic circulation and pulmonary oxidative stress (Brook et al., 2010). At the cellular level, the well-described induction of a pulmonary oxidative stress and inflammation observed after DE, could induce the release of pro-inflammatory mediators that may alter the vascular function (Channell et al., 2012). Several studies have also pointed out the existence of markers of myocardial oxidative stress after DE particulate exposures (Robertson et al., 2014; Yokota et al., 2008). These markers often reflect an early induction of genes involved in the antioxidant response, suggesting rapid adaptive responses (Gurgueira et al., 2002).

More recently, mitochondria have emerged as a target of air pollutants, for example after sulphur dioxide (Qin et al., 2016), carbon monoxide (Reboul et al., 2017) or mining PM (Nichols et al., 2015) exposures. In addition, mitochondrial dysfunction is associated with many cardiovascular diseases, given their central role in a large number of cellular functions such as reactive oxygen species generation and ATP production, as well as apoptosis. Consequently, mitochondria could play a key role in the events leading to cardiovascular diseases due to air pollutants. However, whether diesel exhaust is associated with cardiac mitochondrial dysfunction and/or an oxidative stress and contributes to cardiac dysfunction has not yet been investigated. 
Therefore, the aims of the present study were to test whether a cardiac oxidative stress and/or a mitochondrial defect precede cardiovascular dysfunction after DE exposure and whether the gaseous pollutants contribute to these effects. To achieve these objectives, we exposed animals to dilute exhaust emitted from a diesel motor that was operated under driving cycles, upstream or downstream of a DPF, and after acute or repeated exposures to evaluate immediate and sustained effects.

\section{EXPERIMENTAL PART}

\subsection{Diesel exhaust (DE) generation and emission characterization}

DE were derived from a supercharged common rail direct injection diesel engine equipped with a diesel oxidation catalyst, and located upstream (P1) or downstream (P2) of a DPF, as previously described (Douki et al., 2018). The diesel engine was placed in a test bench cell equipped with a dynamic asynchronous chassis dyno, which allows continuous control of both engine speed and load, as well as the recording of technical parameters. The engine was operated on commercial low sulphur diesel (less than 3 ppm of sulphur), and used under dynamic conditions according to the "New European Driving Cycle" (NEDC). The concentrations of pollutants were monitored as described in the supplementary experimental part and according to previous published methods (Caplain et al., 2006)(Cazier et al., 2016).

\subsection{Exposure study design}

This project was reviewed and approved by a certified committee according to European legislation (authorization number 00291.01). The experiments were performed on adult male Wistar rats (275-300 g, Janvier Inc., Le Genest Saint Isle, France). For inhalation exposures, DE were directly drawn from the exhaust line, directed by a Dekati Fine Particle sampler (FPS) (Dekati Finland) and diluted by a factor 10 as previously described (Douki et al., 2018). Rats were placed in whole body inhalation chambers as previously described (Anselme et al. 2007). All DE exposures were conducted from the exhaust after dilution as described above, 

upstream $(\mathrm{P} 1)$ or downstream $(\mathrm{P} 2)$ of the DPF. For repeated exposures, the animals were exposed for $3 \mathrm{~h} /$ day, 5 days/week during 3 weeks and the biological evaluations were performed after a $16 \mathrm{~h}$ recovery period in clean air. For acute evaluations, rats were exposed for a single $3 \mathrm{~h}$ period and the evaluations were performed after a recovery period of $1 \mathrm{~h}$. This study design is summarized in a scheme presented in the supplementary material and method section.

\subsection{Echocardiographic assessments}

Echocardiographic assessments were performed in sedated rats (100 mg/kg ketamine; 3 mg/kg xylazine; Easote AU5 Advanced Ultrasonography) after the recovery periods. Cardiac ventricular dimensions were measured using M-mode tracings recorded from a 2-dimensional short-axis view at the level of the papillary muscles. Echocardiography provided measurements of LV end-diastolic (LVedd) and end-systolic (LVesd) diameters and posterior wall thickness at diastole (PWEDT) and at systole (PWEST). Relative wall thickness (RWT) was calculated as $2 *$ PWEDT/LVedd. LV systolic function was assessed by the fractional shortening [(LVedd-LVesd)/LVedd] x 100. In addition, velocity-time integral was measured by pulsed-wave Doppler, and cardiac output $(\mathrm{CO})$ was calculated as $\mathrm{CO}=$ aortic velocity-time integral $x\left[\left(\pi \times \mathrm{LV}_{\text {outflow diameter }}\right)^{2} / 4\right] / 100 \times$ heart rate (Moritz et al., 2003).

\subsection{Cardiac mitochondrial assessments}

After echocardiographic assessments, rats were euthanized $(0.2 \mathrm{~g} / \mathrm{kg}$ sodium thiopental $)$, the heart was removed from the chest and the left ventricle (LV) was dissected on ice and weighted. A part of the LV was freshly used for the measurement of mitochondrial oxidative phosphorylation capacity (OXPHOS) and ATP production and the rest was frozen into liquid nitrogen for the mitochondrial enzymatic assays. 
146 We assessed OXPHOS in cardiac permeabilized fibers prepared as described previously

147 (Veksler et al., 1987, Vergeade et al., 2010). Oxygen consumption was measured at $22^{\circ} \mathrm{C}$,

148 using a Clark electrode (Strathkelvin Instruments, Scotland, UK), in a respiration buffer

149 consisting of $2.77 \mathrm{mM} \mathrm{CaK}_{2}$ EGTA (2.77 mM EGTA, 2.77 $\mathrm{mM} \mathrm{CaCO}_{3}$ and $5.54 \mathrm{mM} \mathrm{KOH}$ ),

$1507.23 \mathrm{mM} \mathrm{K} \mathrm{K}_{2}$ EGTA $\left(100 \mathrm{nM}\right.$ free $\left.\mathrm{Ca}^{2+}\right), 1.38 \mathrm{mM} \mathrm{MgCl}\left(1 \mathrm{mM}\right.$ free $\left.\mathrm{Mg}^{2+}\right), 20 \mathrm{mM}$ taurine,

$15190 \mathrm{mM}$ potassium methanesulfonate, $20 \mathrm{mM}$ imidazole, $10 \mathrm{mM}$ sodium methane sulfonate,

152 and $2 \mathrm{mg} / \mathrm{ml} \mathrm{BSA}, \mathrm{pH}$ 7.1. After a stabilization period, $\mathrm{O}_{2}$ consumption rates were recorded

153 with $2 \mathrm{mM}$ ADP, $10 \mathrm{mM}$ glutamate and $4 \mathrm{mM}$ malate as mitochondrial substrate (VGM). To

154 evaluate oxygen consumption from complex II, complex I was blocked with 2 mM amytal

155 and $10 \mathrm{mM}$ succinate were added (VS). Respiration rates are expressed per $\mathrm{mg}$ of proteins of

156 cardiac fibers.

157

ATP production

The ATP production was evaluated from isolated myocardial subsarcolemmal (SSM) and interfibrillar mitochondria (IFM), according to previously described protocols (Palmer et al., 1977; Vergeade et al., 2010) as detailed in the supplementary material and methods. Mitochondrial enzymatic activities

162 The activities of NADH-ubiquinone oxidoreductase (complex I), ubiquinol cytochrome c reductase (complex III), cytochrome c oxidase (complex IV) and citrate synthase activities were assayed in LV homogenates using established methods (Spinazzi et al., 2012) and 165 described in the supplementary material and methods.

\subsection{Parameters of oxidative stress}

167 Cardiac oxidative stress was evaluated from LV homogenates by the measurements of antioxidant enzymes activities and glutathione redox state, as previously described (Moritz et al., 2003). 
171 RNA was isolated from frozen left ventricle (LV) using RNeasy Plus mini kit (Qiagen,

172 France) following manufacture's protocol. Quantity and quality of the RNA were measured

173 using a Nanodrop spectrophotometer and the Agilent 2100 Bioanalyser (Agilent technologies,

174 Santa Clara, CA, USA). RNA with a RNA Integrity Numbers (RIN) higher than 7.8 were

175 used for reverse transcriptase. For hybridization, gene expression was assessed using one chip

176 per LV; 6 replicates from each of the 3 groups exposed to filtered air, repeated exposures of

177 DE upstream (P1) and downstream (P2) DPF, were further processed as follows for

178 GeneChips analysis. The gene expression profiles were determined using GeneChip ${ }^{\circledR}$

179

180

181

182

183

184

185

186

187

188

189

190

191

192

193

194

195

RAGENE 2.0 ST Arrays (> 24000 genes, Affymetrix) through the genomic platform of Hospital Cochin (University Paris Descartes). Samples were hybridized onto array chips, stained, washed, and scanned according to Affymetrix protocol. The array image and cell intensity files (.CEL files) were generated by Affymetrix GeneChip Command Console. After the normalization of the data using global scale normalization, 2 chips were eliminated and further analysis was realized on 28 chips. Resulting signal intensities were on log2-scale.

\subsection{Quantitative real-time PCR}

Total RNA were subjected to reverse transcription using RT Applied Biosystem kit (Courtaboeuf, France). qPCR assays were next performed using Power SYBR Green PCR master kit according to the manufacturer's instructions (Life Technologies) and an ABI 7900 detector (Applied Biosystem). Kicqstart gene-specific primers were purchased from SigmaAldrich (St Quentin Fallavier, France). Amplification curves of the PCR products were analyzed with the ABI Prism SDS software using the comparative cycle threshold method.

The relative gene expression was calculated by using the $\Delta \Delta C_{T}$ analysis for each sample after normalization against $\beta$-actin gene expression. The control air-exposed rats served as a reference and their mRNA expression was arbitrarily considered as 1 unit for each analyzed gene. 
197 For biological parameters, results are expressed as mean \pm sem. One-way ANOVA was used 198 to compare the effects of DE exposures, followed by the post-hoc Tukey test where 199 appropriate (normal distribution verified by the Shapiro-Wilk test) or by Kruskal-Wallis test, 200 followed by Dunn's multiple comparison post-test. Differences were considered statistically 201 significant when $\mathrm{p}<0.05$. 
3. RESUlts

\subsection{Diesel exhaust characterization}

204 The exhaust characterization was performed from raw exhaust in both upstream (P1) and 205 downstream (P2) of the DPF, during the NEDC cycles and the results for regulated pollutants were presented in the Table S1. The DPF reduced the total PM concentrations (P2). Cold start induced high emissions of $\mathrm{CO}$ and total gaseous hydrocarbons whereas $\mathrm{NO}_{2}$ emissions were increased during driving conditions. Based on these measurements, we consider that after dilution, the concentrations of pollutants to which rats were exposed in P1 were $2.5 \mathrm{mg}$ $210 \mathrm{PM} / \mathrm{m}^{3}$ for the mean with a median at $1.5 \mathrm{mg} / \mathrm{m}^{3}$. For $\mathrm{NO}_{2}$, the rats were exposed to an average level of 3 ppm after the first NEDC cycle.

212 Concentrations of aldehydes and mono-aromatic volatile organic compounds (VOCs) were measured two times, on two different days randomly selected during the study (Table S2).

214 The total aldehyde concentrations measured upstream and downstream of the DPF are quite 215 homogeneous during the two days. Formaldehyde and acetaldehyde are the main carbonyl compounds detected. An increase by a 4-fold factor of the total carbonyl compound concentration is noted downstream of the DPF (P2). The total mono-aromatic VOC concentration decreases by a ten-fold downstream of the DPF (P2) (Table S2).

219 For alkanes (Fig. S1) and PAH (Fig. S2), a decrease of the total concentrations is observed in 220 P2. The main alkanes are between $\mathrm{C} 15$ and C30 in P1, whereas in P2 the concentrations of 221 alkanes heavier than C25 strongly decrease (Fig. S1). Naphtalene represents more than $80 \%$ 222 of the detected PAH and shows a strong decrease (nearly 70\%) after the DPF. The other PAH 223 detected are phenanthrene, fluoranthene and pyrene. No heavier PAH is detected due to the 224 new engine generation with high pressure injection, which optimizes the fuel combustion and 225 thus decreases the heavy PAH generation (Fig S2). 
3.2. Cardiovascular function following diesel exposure.

228 After acute DE exposure, a slight but significant increase in both LV end-diastolic (+5, $\mathrm{p}<0.05$ and $+8 \%, \mathrm{p}<0.01, \mathrm{P} 1$ and $\mathrm{P} 2$ respectively, vs. control $)$ and end-systolic diameters $(+16$ and $+18 \%, \mathrm{p}<0.05 \mathrm{P} 1$ and $\mathrm{P} 2$ respectively, vs. control) was observed (Fig 1). This increase translates a limited dilation of ventricular chambers. This impairment persisted and worsened 232 after repeated exposures, with an increase of about $30 \%$ in both LV end-diastolic $(\mathrm{p}<0.001)$ 233 and end-systolic $(\mathrm{p}<0.01)$ diameters vs. control and a decrease in fractional shortening by $23415 \%$ downstream of the DPF, whereas cardiac output was not modified (Fig. 1). Rats exposed 235 to DE also exhibited a decrease in posterior wall thickness at systole and diastole after 3 236 weeks of exposure (Table S3).
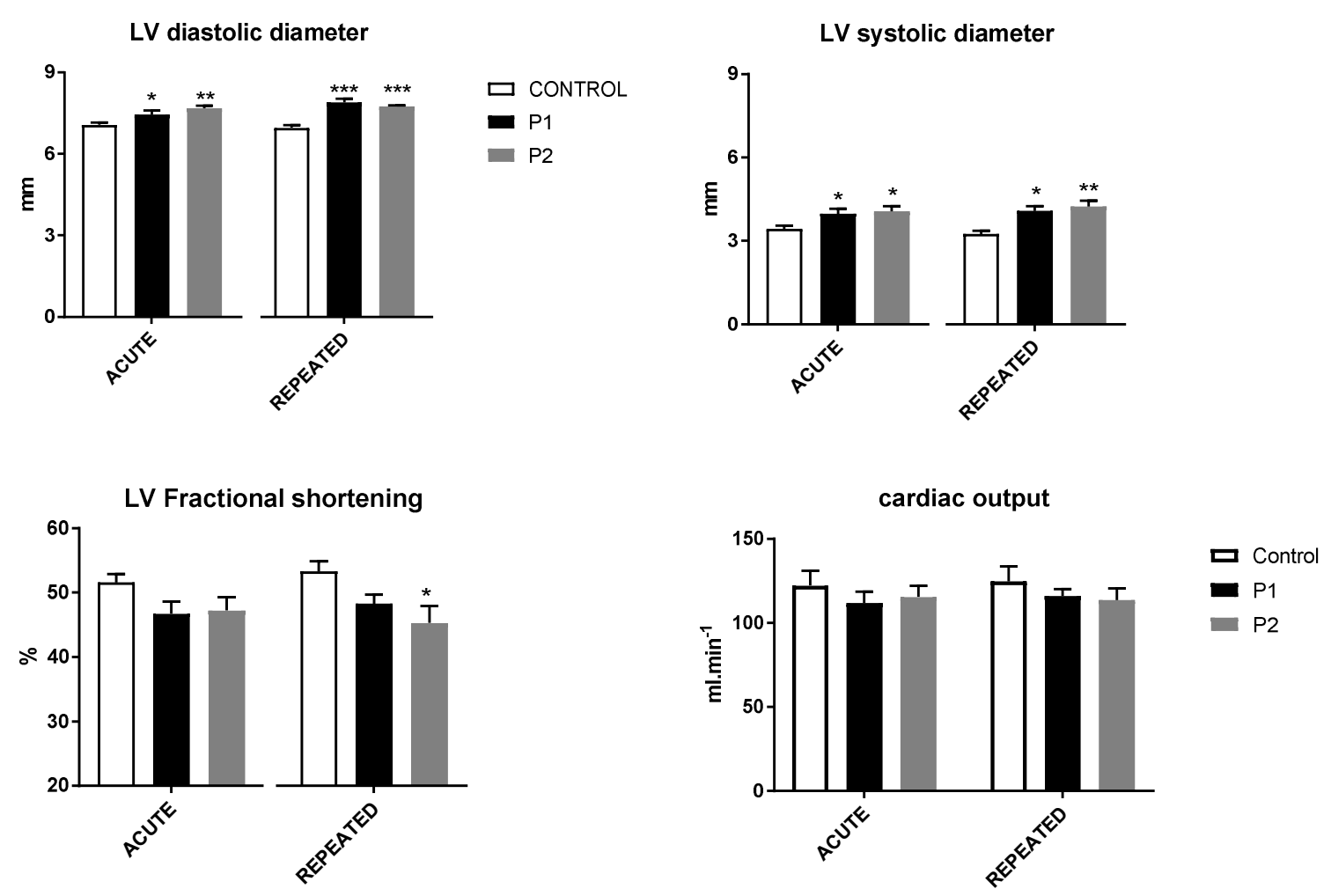

238 Figure 1: Effect of DE on echocardiographic parameters after acute or repeated exposures.

239 Rats were exposed to DE derived upstream (P1) or downstream (P2) of the DPF. * p $<0.05$, $* * \mathrm{p}<0.01, * * * \mathrm{p}<0.001$ vs Control (n $=6-10$ rats) 
A

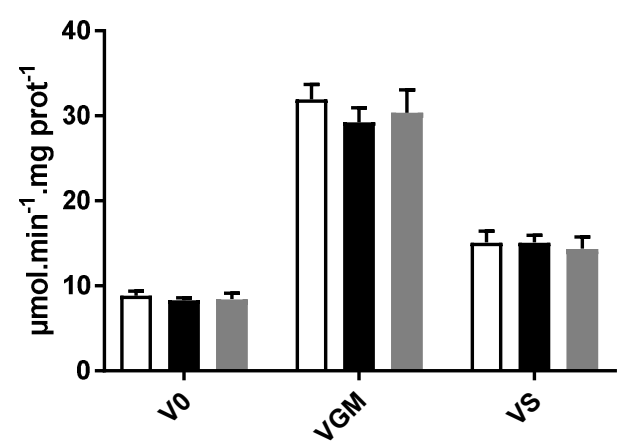

B

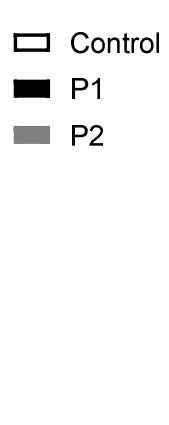

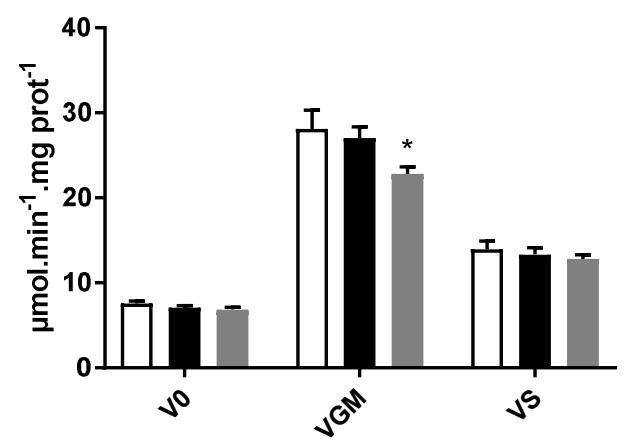

Figure 2: Respiration in LV skinned cardiac fibers after acute or repeated exposures. Basal respiration rate (V0), complex I respiration with glutamate and malate (VGM), complex II respiration with succinate (VS) were evaluated in Control, P1 and P2 groups, after acute (A) or repeated exposures $(\mathrm{B}) .{ }^{*} \mathrm{p}<0.05$ vs Control ( $\mathrm{n}=6-10$ rats in duplicate).

To further investigate the effects of repeated DE exposure on mitochondrial OXPHOS, the functional activities of mitochondrial respiratory chain complexes I, III and IV were analyzed 
DPF, compared with the control group, whereas no significant changes were found for

261 complex III or complex IV activities. Activity of citrate synthase, a mitochondrial-specific matrix protein, was not significantly different between the groups.
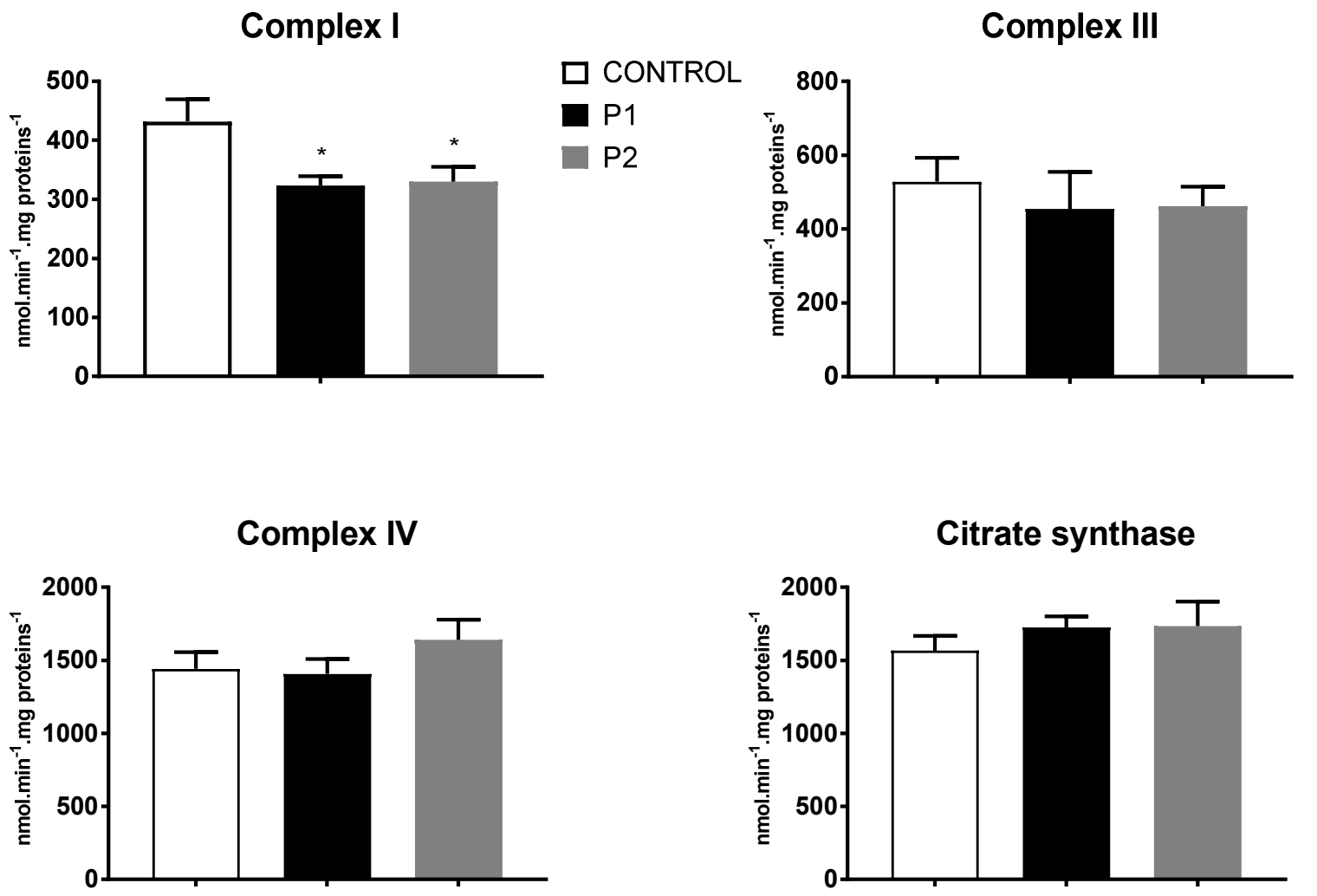

Figure 3: Mitochondrial enzymatic activities in LV after repeated exposures. Enzymatic activities were evaluated in LV homogenates from Control, P1 and P2 groups. *p<0.05 vs. Control ( $\mathrm{n}=8$ rats) To complete the assessment of mitochondrial function, we next measured ATP production rates in freshly isolated mitochondrial fractions, of exposed rats during 3 weeks. For this evaluation, we performed the isolation of SSM and IFM after proteinase treatment. We found that ATP production was reduced specifically in the IFM fractions after repeated DE exposure

271 compared with the control group, whereas there was no change in ATP production in the SSM 272 fractions (Fig. S3). 
274

275

276

277

278

279

280

A wide genomic analysis of DE effects on LV was performed considering a p-value LogRatio $<0.05$. Figure 4 represents the number of genes regulated after repeated exposure of DE. It reveals that 1921 and 970 genes were identified as regulated in P1 vs Control and P2 vs Control, respectively, thus showing that the presence of DPF reduces by half the number of genes regulated by DE. Among the DE-regulated genes, we found that about $50 \%$ of genes are up- or down-regulated both for P1 vs. Control and P2 vs. Control (Fig.4). Venn diagram also reveals that only 178 responsive genes are common to P1 and P2, when compared to control, corresponding to $9.2 \%$ and $18.3 \%$ of genes from P1 vs. Control and P2 vs. Control, respectively.

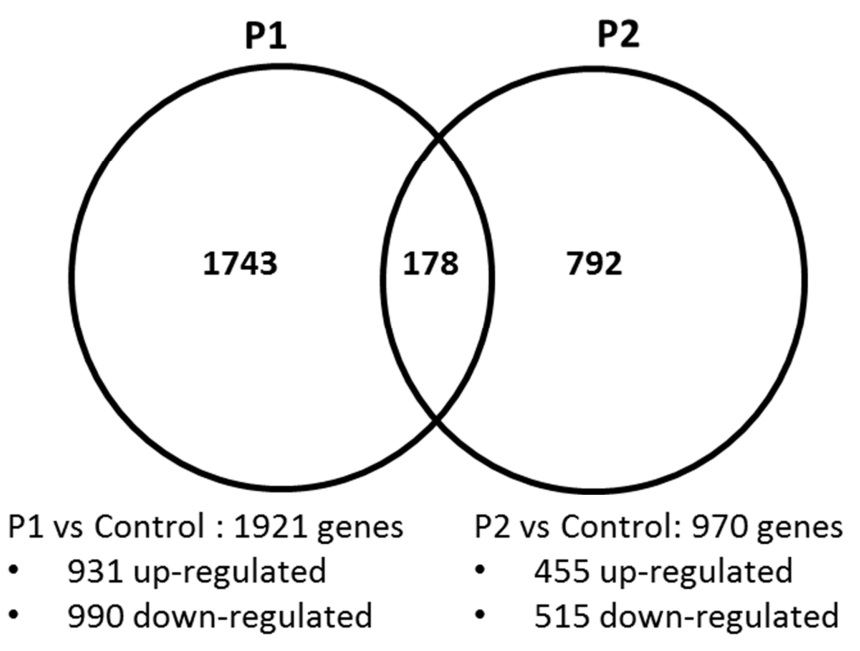

Figure 4: Number of DE-regulated genes detected by microarray in LV after repeated exposure. Venn diagrams illustrate the number of genes regulated in P1 and P2 when compared to control. Genes were selected based on a pValue LogRatio $<0.05$ ( $n=6$ rats)

The lists of genes corresponding to P1 vs Control (1921 genes), to P2 vs Control (970 genes) and to genes in common in both groups (178 genes) can be found as supplementary tables (Tables excel S5, S6 and S7). As previously described by our laboratory on lung tissue after repeated exposure to DE (Douki et al, 2018), a high proportion of genes identified as DE- 
responsive corresponds in fact to slightly regulated genes. Thus, after applying a cut-off at 1.3

293 fold change factor to avoid the impact of differentially expressed genes with very small change (less than 1.3-fold), we found that the numbers of genes regulated by DE vs control were 234 (representing $12.1 \%$ of 1921 genes) and 46 (representing $4.7 \%$ of 970 genes) for exposure conditions upstream (P1) and downstream (P2) of DPF, respectively (Tables S5 and S6).

Concerning the common genes, we identified that 21 genes were regulated by a factor $\geq 1.3$ fold and, among them, 10 genes were up-regulated and 11 genes were down-regulated by DE exposure (Table S7).

Next, we have submitted to the Ingenuity Pathway Analysis (IPA) software (Ingenuity Systems, Mountain View, CA) the P1 (1921 genes) and the P2 (970 genes) gene lists. The 5 top canonical pathways and the 5 top Tox lists for P1 vs Control and P2 vs Control are represented in the Table S8. IPA analysis reveals that pathways related to "mitochondria" appear several times in the P1 group vs. control (mitochondrial dysfunction, Increases permeability transition of mitochondria and mitochondrial membrane and Oxidative phosphorylation) and one time in the P2 group vs. Control (Swelling of mitochondria), demonstrating the importance of DE effects on mitochondria in LV tissue. Among the genes from the mitochondrial dysfunction and Oxidative phosphorylation pathway, several corresponded to genes coding for mitochondrial complex I subunits (.i.e. NDUFA7, NDUFB6, NDUFC1...); therefore, we analyzed the level of expression of some of these genes by qPCR. Our results showed that NDUFA7 mRNA expression was significantly upregulated in $\mathrm{LV}$ from rats exposed to $\mathrm{DE}$ in $\mathrm{P} 1$ when compared to control rats and to rats exposed to DE in P2 (Fig.5). By contrast, the levels of mRNA expression of genes coding for other subunits of mitochondrial complex I were not significantly modified by repeated DE exposure (Fig.5). Such an absence of significance may be related to the dispersion of mRNA 
expression of these genes observed in the group of rats exposed to DE in P1 and also, to the

318 weak modulation factors found in microarrays, ranging between -2.1 to 1.9 fold, as previously

319 described in a similar study realized on lung tissue after DE repeated exposures (Douki et al., 320 2018).

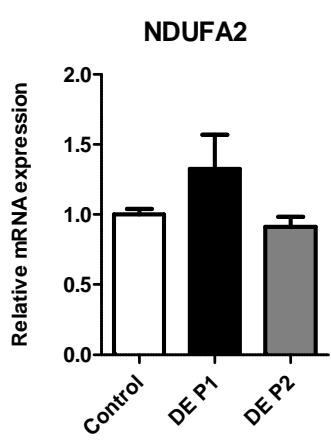

NDUFB2

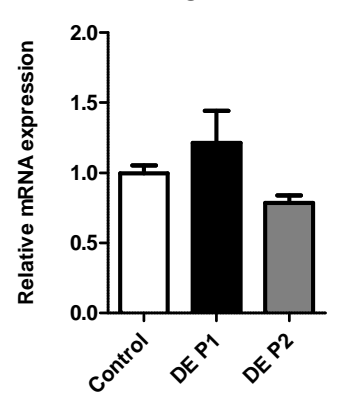
$<0.05$ and $* * \mathrm{p}<0.01$
NDUFA6

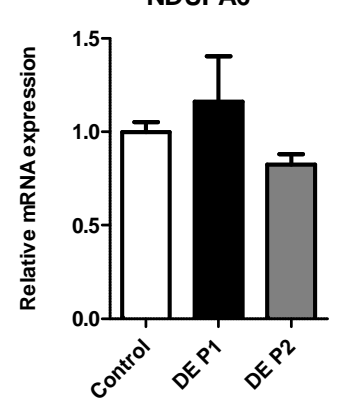

NDUFB6

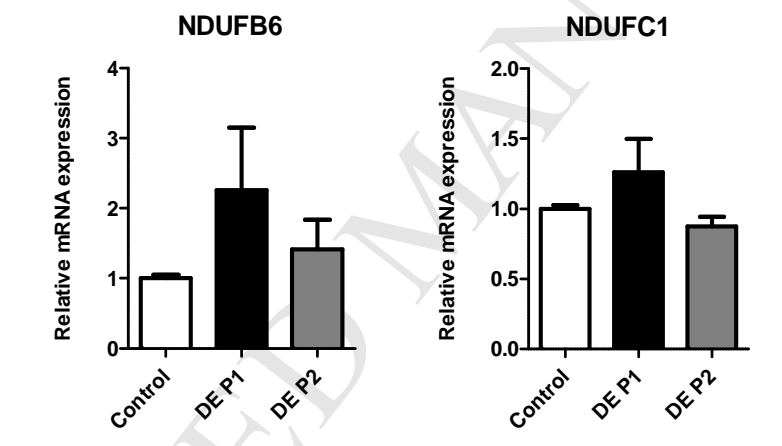

NDUFA7

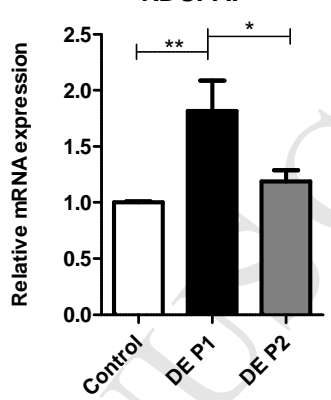

NDUFC1

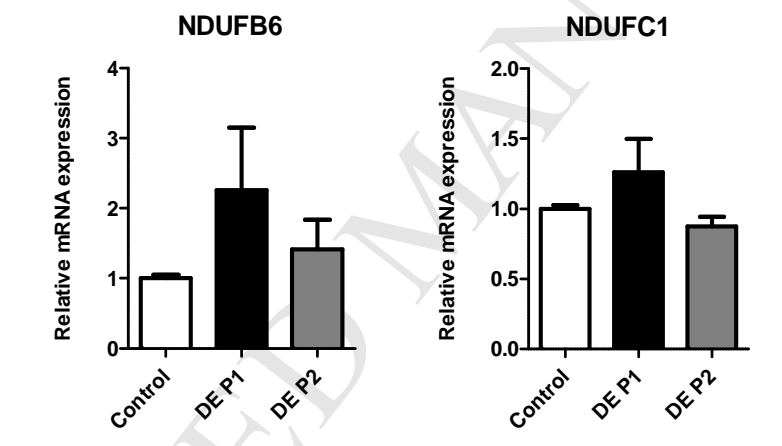

NDUFAF4

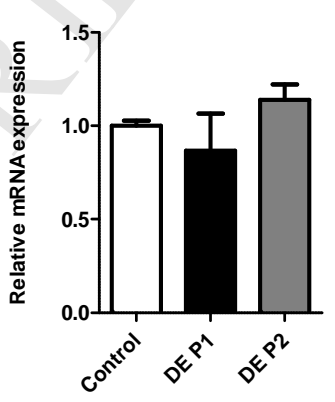

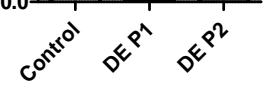

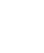

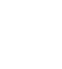

Figure 5: Expression analysis of genes coding for the mitochondrial complex I. mRNA levels were determined by RT-qPCR. Data are expressed relatively to mRNA levels found in the Control group exposed to air and arbitrarily set at the value of $1 . \mathrm{N}=6$, Means \pm SEM. $* \mathrm{p}$ 
In this study, we compared cardiac effects after exposure with exhaust collected downstream (P2) or upstream (P1) of a DPF using a standard diesel and engine, representative of the current fleet and used under dynamic conditions to mimic emissions produced in urban driving. In such conditions, we provide evidence that these DE produce a sustained cardiac and mitochondrial dysfunction associated with an oxidative stress-independent impairment of the mitochondrial function after 3 weeks of exposure in healthy rats.

Many conditions may affect diesel vehicle emissions, such as driving conditions, posttreatment technologies that reduce regulatory pollutant emissions, or fuel composition. In this study, the urban cold start driving condition produces the highest emissions of total gaseous hydrocarbons and $\mathrm{CO}$, whereas these emissions are below the limit for detection during the other driving conditions, due to the catalysis. However, oxidation catalysis causes an increase in $\mathrm{NO}_{2}$ emissions, observed during the driving conditions. These observations are in agreement with Martinet et al (2017) (Martinet et al., 2017) and these elevated $\mathrm{NO}_{2}$ emissions may contribute to the biological effects of DE, as previously suggested (Karthikeyan et al., 2013). Note that the $\mathrm{NO}_{2}$ levels in our study are similar between $\mathrm{P} 1$ and $\mathrm{P} 2$ due to the diesel oxidation catalyst located upstream to regenerate the DPF with $\mathrm{NO}_{2}$, an active oxidation agent used in the regeneration process of the soot accumulated in the DPF (Kandylas and Koltsakis, 2002). Particle filtration significantly reduces particle mass emission, with a near $100 \%$ efficiency associated to a decrease in alkane and PAH levels, as well as VOCs such as BTEX. However, carbonyl compounds, acetaldehyde and formaldehyde, emissions remain abundant and the levels increase after the DPF. These results indicate that the particle trap may have changed the composition of exhaust, but additional analyses are required to confirm it, even though a previous study led to similar observations (Ratcliff et al., 2010). 
In these experimental conditions, with or without DPF, our results suggest that repeated DE exposures result in cardiac dysfunction. Indeed, the significant increases in LV diastolic and systolic diameters observed, show LV dilatation associated with reduced LV contractility, but LV dysfunction is only moderate since cardiac output is not modified. These results indicate that LV remodeling is sufficient to maintain global cardiac function. However, it should be outlined that our experimental conditions probably underestimate the deleterious effects of repeated DE exposure. Indeed, LV function was only determined 16-18 $\mathrm{h}$ after the last exposure, excluding the deleterious direct effects of DE, as observed $1 \mathrm{~h}$ after acute DE exposure. Furthermore, it is tempting to state that if LV function had been measured $1 \mathrm{~h}$ after the last exposure of the repeated DE exposure, LV dysfunction would have been more marked, since it would combine both acute direct as well as chronic adaptive mechanisms.

372 Similarly, one might question whether longer repeated DE exposure would have induced 373 more marked cardiac dysfunction.

Moreover, our results clearly show the importance of gaseous phase vs. particles since both $\mathrm{P} 1$, i.e. whole exhaust, and $\mathrm{P} 2$, i.e. gaseous phase without particles, induce a similar increases in LV diastolic/systolic diameters after either single or repeated DE exposure. Consequently, our study expands the knowledge of DE's deleterious effects on health, since, as shown 378 previously, DE particles alone decrease fractional shortening and induce end-diastolic 379 diameters after repeated exposures in healthy rats (Bradley et al., 2013).

The mechanisms underlying the cardiovascular effects of DE remain only partially 381 understood, but a systemic inflammatory response associated with an oxidative stress initiated 382 in the lung may relate to the triggering of the cardiovascular effects (Brook et al., 2010; 383 Nemmar et al., 2018). Our laboratory previously observed a limited accumulation of oxidative 384 damage in the lungs following repeated exposure to DE (Douki et al., 2018). Consistent with these previous findings, our data provide no evidence of a cardiac oxidative stress, as cardiac 
redox parameters remain unchanged. Indeed, neither cardiac antioxidant enzymes, nor reduced/oxidized glutathione ratio, were significantly modified after acute and repeated exposures. We also measured activity of aconitase, a sensitive mitochondrial enzyme of superoxide (Gardner, 2002), but observed no differences. Even though we cannot rule out a brief effect of reactive oxygen species, oxidative stress does not seem to be a major triggering event for the cardiovascular effects observed in this study. DE-related oxidative stress is often attributable to PM and related to their physico-chemical characteristics, in particular surface bound organic compounds such as metals, quinones and PAH (Charrier and Anastasio, 2012;

Cho et al., 2005; Crobeddu et al., 2017). The concentration of these particulate constituents evaluated in this study is lower, as previously evaluated in new diesel engines than in traditional DE (McClellan et al., 2012); this may explain the lack of a sustained oxidative stress in this study.

To identify the cellular mechanisms contributing to cardiac dysfunction following DE exposures, we next focused on mitochondrial function, the primary source of energy in the myocardium. For this purpose, we first evaluated oxygen consumption in situ, in saponinskinned cardiac fibers that ensure global mitochondrial function assessment in intact mitochondria (Veksler et al., 1987). We showed that respiration rate with complex I-linked substrates (glutamate and malate) was affected in the hearts of rats exposed to repeated 404 filtered DE. However, when succinate was provided as substrate, mitochondrial respiration was not affected, suggesting that the electron chain was not affected downstream of the complex II. As we did not observe any mitochondrial dysfunction after an acute exposure, we next performed further mitochondrial investigations in the hearts of rats exposed to repeated DE and observed a decrease in complex I activity specifically. Indeed, the tissue activities of 409 complexes III and IV, and citrate synthase were not modified. Taken together, these results indicated that decreased respiration rates with glutamate and malate are due to decrease in 
411

412

413

414

complex I function. Complex I dysfunction has also been observed in several diseases including heart failure (Scheubel et al., 2002), ischemia-reperfusion (Kang et al., 2018), or during chronic cardiac pressure overload (Schrepper et al., 2012). DE can instigate adverse cardiovascular response by activation of the sympathetic nervous system mediated through activation of pulmonary sensory receptors and adrenergic receptors (Robertson et al., 2014). This activation is associated with elevated blood pressure and increases vulnerability to ischemia and reperfusion injury (Robertson et al., 2014). Although the underlying molecular mechanisms connecting cardiac mitochondrial complex I defect and pulmonary exposure to DE remain to be deciphered, this mitochondrial dysfunction could participate, at least partially, in this vulnerability after DE exposure.

The impact on mitochondria of repeated exposure to DE was further investigated by transcriptional analysis of LV tissue. Several pathways relating to mitochondria were identified in LV tissue from exposed rats, such as "oxidative phosphorylation" and "mitochondrial dysfunction" (P1 vs. Control) or "swelling of mitochondria" (P2 vs. Control), indicating the impact of the DE exposures on the mitochondria. Individual gene expression analysis revealed that very few genes were significantly regulated by DE exposure, probably due to an adaptive response after exposures, since these results were obtained after 3 weeks of repeated exposures, with $16 \mathrm{~h}$ post-exposure. In these conditions, our results showed that NDUFA7 mRNA expression was slightly but significantly up-regulated in rats exposed to DE and a similar trend was observed for NDUFB6. These genes encode subunits of NADH:ubiquinone oxidoreductase (complex I) and this result indicates that the changes in complex I activity may not be due to a decrease in gene expression but may reflect an adaptive mechanism in response to the decrease in complex I activity. Another regulatory pattern for changes in respiratory complex activities may be considered such as impaired 
assembly of subunits to supercomplexes (Rosca et al., 2008) but further investigations are needed.

437 Complex I is the largest multi-subunit complex of the respiratory chain and is one of the 438 complexes that generate the proton-motive force required for ATP synthesis. In order to 439 evaluate the consequences on the synthesis of ATP, we evaluated ATP production in two 440 isolated mitochondrial subpopulations, subsarcolemmal (SSM) and interfibrillar mitochondria 441 (IFM), and observed a decrease in ATP synthesis capacity selectively in IFM. As described in 442 the literature, subpopulations of mitochondria are structurally and metabolically distinct and 443 are differently susceptible to pathological stimuli (Hollander et al., 2014). The defect in 444 mitochondrial ATP synthesis observed in the IFM population is also consistent with the 445 known susceptibility of these mitochondrial subpopulations to heart failure (Schwarzer et al., 446 2013) or aging (Hofer et al., 2009). This result is clinically relevant as the heart is particularly 447 vulnerable to limited ATP supply because of its large energy request.

448 It is noteworthy that these mitochondrial changes are observed with whole DE (P1, mRNA 449 expression), filtered DE (P2, oxygen consumption), or both (ATP synthesis and complex I 450 activity). These apparent discrepancies could be explained by methodological differences. 451 Within the respiratory chain, $\mathrm{O}_{2}$ consumption measured from LV skinned cardiac fibers makes it possible to maintain the interactions of assembled complexes and electron transporters, which influence the measurement of respiratory capacity, whereas complex activity as well as ATP synthesis capacity are independent of these influences. These results 455 might also translate the relative effect of the particle and gas phases and their different interaction with the mitochondria. Whole DE contains respirable soot-particles, but although 457 the cardiovascular effects of particles have been extensively explored, few studies attempted 458 to estimate the mitochondrial function in the heart. A previous study performed in mice showed a time-dependent and reversible decrease in $\mathrm{O}_{2}$ consumption after acute residual oil 
460

461

462

463

464

fly ash (ROFA) (Marchini et al., 2013). Though restricted to an acute ROFA exposure, this result showed an impairment of mitochondrial function associated with deficient cardiac contractility. With regard to gaseous phase, some combustion-related gases, such as $\mathrm{CO}$ (Reboul et al., 2017) and $\mathrm{SO}_{2}$ (Qin et al., 2016), have previously shown to induce mitochondrial effects. However, it remains difficult to connect an effect in response to a specific pollutant in a complex mixture, for which the composition varies according to the driving conditions and after treatment. Notwithstanding these limitations, the findings of the present study are new and suggest that the mitochondrial impairment contributes to the clinical cardiovascular events observed after DE exposure.

In conclusion, this study performed with diluted DE emitted from an engine used under dynamic conditions revealed sustained cardiovascular and mitochondrial effects attributable to the gas phase, possibly aldehydes and/or $\mathrm{NO}_{2}$, but further study is warranted to clarify the precise role of these pollutants. Although the predictivity of these results to humans remains delicate due to limitations inherent in rodent studies, the effects observed in cardiac mitochondria may suggest significant consequences in terms of cardiac effects for vulnerable populations with underlying energy deficit such as patients with heart failure or the elderly.

\section{Conflict of interest statement}

Authors declare that there are no conflicts of interest.

\section{Funding}

This work was supported by ADEME (French Environment and Energy Management Agency; Cardiox project, grant number 2013-1-237), Inserm (grant number ENV201207) and the Regional Council of Haute-Normandie.

\section{Acknowledgement}


483 The authors would like to thank P. Scotti di Vettimo and Dr N. Theret for their advice in 484 bioinformatics analysis. 
Bradley, J.M., Cryar, K.A., El Hajj, M.C., El Hajj, E.C., and Gardner, J.D. (2013). Exposure to diesel exhaust particulates induces cardiac dysfunction and remodeling. J. Appl. Physiol. 115, 10991106.

Brook, R.D., Rajagopalan, S., Pope, C.A., Brook, J.R., Bhatnagar, A., Diez-Roux, A.V., Holguin, F., Hong, Y., Luepker, R.V., Mittleman, M.A., et al. (2010). Particulate matter air pollution and cardiovascular disease: An update to the scientific statement from the American Heart Association. Circulation 121, 2331-2378.

Burnett, R., Chen, H., Szyszkowicz, M., Fann, N., Hubbell, B., Pope, C.A., Apte, J.S., Brauer, M., Cohen, A., Weichenthal, S., et al. (2018). Global estimates of mortality associated with long-term exposure to outdoor fine particulate matter. Proc. Natl. Acad. Sci. U.S.A. 115, 9592-9597.

Caplain, I., Cazier, F., Nouali, H., Mercier, A., Déchaux, J.-C., Nollet, V., Joumard, R., André, J.-M., and Vidon, R. (2006). Emissions of unregulated pollutants from European gasoline and diesel passenger cars. Atmospheric Environment 40, 5954-5966.

Carll, A.P., Hazari, M.S., Perez, C.M., Krantz, Q.T., King, C.J., Winsett, D.W., Costa, D.L., and Farraj, A.K. (2012). Whole and particle-free diesel exhausts differentially affect cardiac electrophysiology, blood pressure, and autonomic balance in heart failure-prone rats. Toxicol. Sci. 128, 490-499.

Cazier, F., Genevray, P., Dewaele, D., Nouali, H., Verdin, A., Ledoux, F., Hachimi, A., Courcot, L., Billet, S., Bouhsina, S., et al. (2016). Characterisation and seasonal variations of particles in the atmosphere of rural, urban and industrial areas: Organic compounds. Journal of Environmental Sciences 44, 45-56.

Channell, M.M., Paffett, M.L., Devlin, R.B., Madden, M.C., and Campen, M.J. (2012). Circulating factors induce coronary endothelial cell activation following exposure to inhaled diesel exhaust and nitrogen dioxide in humans: evidence from a novel translational in vitro model. Toxicol. Sci. $127,179-186$.

Charrier, J.G., and Anastasio, C. (2012). On dithiothreitol (DTT) as a measure of oxidative potential for ambient particles: evidence for the importance of soluble transition metals. Atmos Chem Phys 12, 11317-11350.

Cho, A.K., Sioutas, C., Miguel, A.H., Kumagai, Y., Schmitz, D.A., Singh, M., Eiguren-Fernandez, A., and Froines, J.R. (2005). Redox activity of airborne particulate matter at different sites in the Los Angeles Basin. Environ. Res. 99, 40-47.

Conklin, D.J., Kong, M., and HEI Health Review Committee (2015). Part 4. Assessment of plasma markers and cardiovascular responses in rats after chronic exposure to new-technology diesel exhaust in the ACES bioassay. Res Rep Health Eff Inst 111-139; discussion 141-171.

Crobeddu, B., Aragao-Santiago, L., Bui, L.-C., Boland, S., and Baeza Squiban, A. (2017). Oxidative potential of particulate matter 2.5 as predictive indicator of cellular stress. Environ. Pollut. 230, $125-133$.

Douki, T., Corbière, C., Preterre, D., Martin, P.J., Lecureur, V., André, V., Landkocz, Y., Pottier, I., Keravec, V., Fardel, O., et al. (2018). Comparative study of diesel and biodiesel exhausts on lung oxidative stress and genotoxicity in rats. Environ. Pollut. 235, 514-524.

Folino, F., Buja, G., Zanotto, G., Marras, E., Allocca, G., Vaccari, D., Gasparini, G., Bertaglia, E., Zoppo, F., Calzolari, V., et al. (2017). Association between air pollution and ventricular 
arrhythmias in high-risk patients (ARIA study): a multicentre longitudinal study. Lancet Planet

528 Health 1, e58-e64.

529 Gardner, P.R. (2002). Aconitase: sensitive target and measure of superoxide. Meth. Enzymol. 349,

\section{9-23.}

531

532

533

534

535

536

537

538

539

540

541

542

543

544

545

546

547

548

549

550

551

552

553

554

555

556

557

558

559

560

561

562

563

564
Gurgueira, S.A., Lawrence, J., Coull, B., Murthy, G.G.K., and González-Flecha, B. (2002). Rapid increases in the steady-state concentration of reactive oxygen species in the lungs and heart after particulate air pollution inhalation. Environ. Health Perspect. 110, 749-755.

Hofer, T., Servais, S., Seo, A.Y., Marzetti, E., Hiona, A., Upadhyay, S.J., Wohlgemuth, S.E., and Leeuwenburgh, C. (2009). Bioenergetics and permeability transition pore opening in heart subsarcolemmal and interfibrillar mitochondria: effects of aging and lifelong calorie restriction. Mech. Ageing Dev. 130, 297-307.

Hollander, J.M., Thapa, D., and Shepherd, D.L. (2014). Physiological and structural differences in spatially distinct subpopulations of cardiac mitochondria: influence of cardiac pathologies. Am. J. Physiol. Heart Circ. Physiol. 307, H1-14.

Honda, T., Pun, V.C., Manjourides, J., and Suh, H. (2018). Associations of long-term fine particulate matter exposure with prevalent hypertension and increased blood pressure in older Americans. Environ. Res. 164, 1-8.

Ibald-Mulli, A., Stieber, J., Wichmann, H.E., Koenig, W., and Peters, A. (2001). Effects of air pollution on blood pressure: a population-based approach. Am J Public Health 91, 571-577.

Kandylas, I.P., and Koltsakis, G.C. (2002). NO2-Assisted Regeneration of Diesel Particulate Filters: A Modeling Study. Ind. Eng. Chem. Res. 41, 2115-2123.

Kang, P.T., Chen, C.-L., Lin, P., Zhang, L., Zweier, J.L., and Chen, Y.-R. (2018). Mitochondrial complex I in the post-ischemic heart: reperfusion-mediated oxidative injury and protein cysteine sulfonation. J. Mol. Cell. Cardiol. 121, 190-204.

Karthikeyan, S., Thomson, E.M., Kumarathasan, P., Guénette, J., Rosenblatt, D., Chan, T., Rideout, G., and Vincent, R. (2013). Nitrogen dioxide and ultrafine particles dominate the biological effects of inhaled diesel exhaust treated by a catalyzed diesel particulate filter. Toxicol. Sci. 135, 437-450.

Krishnan, R.M., Adar, S.D., Szpiro, A.A., Jorgensen, N.W., Van Hee, V.C., Barr, R.G., O’Neill, M.S., Herrington, D.M., Polak, J.F., and Kaufman, J.D. (2012). Vascular responses to long- and shortterm exposure to fine particulate matter: MESA Air (Multi-Ethnic Study of Atherosclerosis and Air Pollution). J. Am. Coll. Cardiol. 60, 2158-2166.

Landrigan, P.J., Fuller, R., Acosta, N.J.R., Adeyi, O., Arnold, R., Basu, N.N., Baldé, A.B., Bertollini, R., Bose-O'Reilly, S., Boufford, J.I., et al. (2018). The Lancet Commission on pollution and health. Lancet 391, 462-512.

Link, M.S., Luttmann-Gibson, H., Schwartz, J., Mittleman, M.A., Wessler, B., Gold, D.R., Dockery, D.W., and Laden, F. (2013). Acute exposure to air pollution triggers atrial fibrillation. J. Am. Coll. Cardiol. 62, 816-825.

Lucking, A.J., Lundbäck, M., Barath, S.L., Mills, N.L., Sidhu, M.K., Langrish, J.P., Boon, N.A., Pourazar, J., Badimon, J.J., Gerlofs-Nijland, M.E., et al. (2011). Particle traps prevent adverse vascular and prothrombotic effects of diesel engine exhaust inhalation in men. Circulation 123, 1721-1728. 
Marchini, T., Magnani, N., D’Annunzio, V., Tasat, D., Gelpi, R.J., Alvarez, S., and Evelson, P. (2013). Impaired cardiac mitochondrial function and contractile reserve following an acute exposure to environmental particulate matter. Biochim. Biophys. Acta 1830, 2545-2552.

Martinet, S., Liu, Y., Louis, C., Tassel, P., Perret, P., Chaumond, A., and André, M. (2017). Euro 6 Unregulated Pollutant Characterization and Statistical Analysis of After-Treatment Device and Driving-Condition Impact on Recent Passenger-Car Emissions. Environ. Sci. Technol. 51, 58475855.

McClellan, R.O., Hesterberg, T.W., and Wall, J.C. (2012). Evaluation of carcinogenic hazard of diesel engine exhaust needs to consider revolutionary changes in diesel technology. Regul. Toxicol. Pharmacol. 63, 225-258.

Mordukhovich, I., Coull, B., Kloog, I., Koutrakis, P., Vokonas, P., and Schwartz, J. (2015). Exposure to sub-chronic and long-term particulate air pollution and heart rate variability in an elderly cohort: the Normative Aging Study. Environ Health 14, 87.

Moritz, F., Monteil, C., Mulder, P., Derumeaux, G., Bizet, C., Renet, S., Lallemand, F., Richard, V., and Thuillez, C. (2003). Prolonged cardiac dysfunction after withdrawal of chronic cocaine exposure in rats. J. Cardiovasc. Pharmacol. 42, 642-647.

Nemmar, A., Hoet, P.H.M., Dinsdale, D., Vermylen, J., Hoylaerts, M.F., and Nemery, B. (2003). Diesel exhaust particles in lung acutely enhance experimental peripheral thrombosis. Circulation 107, 1202-1208.

Nemmar, A., Al-Salam, S., Beegam, S., Yuvaraju, P., and Ali, B.H. (2018). Thrombosis and systemic and cardiac oxidative stress and DNA damage induced by pulmonary exposure to diesel exhaust particles and the effect of nootkatone thereon. Am. J. Physiol. Heart Circ. Physiol. 314, H917H927.

Nichols, C.E., Shepherd, D.L., Knuckles, T.L., Thapa, D., Stricker, J.C., Stapleton, P.A., Minarchick, V.C., Erdely, A., Zeidler-Erdely, P.C., Alway, S.E., et al. (2015). Cardiac and mitochondrial dysfunction following acute pulmonary exposure to mountaintop removal mining particulate matter. Am. J. Physiol. Heart Circ. Physiol. 309, H2017-2030.

Palmer, J.W., Tandler, B., and Hoppel, C.L. (1977). Biochemical properties of subsarcolemmal and interfibrillar mitochondria isolated from rat cardiac muscle. J. Biol. Chem. 252, 8731-8739.

Pope, C.A., Bhatnagar, A., McCracken, J.P., Abplanalp, W., Conklin, D.J., and O’Toole, T. (2016). Exposure to Fine Particulate Air Pollution Is Associated With Endothelial Injury and Systemic Inflammation. Circ. Res. 119, 1204-1214.

Puett, R.C., Hart, J.E., Yanosky, J.D., Paciorek, C., Schwartz, J., Suh, H., Speizer, F.E., and Laden, F. (2009). Chronic fine and coarse particulate exposure, mortality, and coronary heart disease in the Nurses' Health Study. Environ. Health Perspect. 117, 1697-1701.

Qin, G., Wu, M., Wang, J., Xu, Z., Xia, J., and Sang, N. (2016). Sulfur Dioxide Contributes to the Cardiac and Mitochondrial Dysfunction in Rats. Toxicol. Sci. 151, 334-346.

Ratcliff, M.A., Dane, A.J., Williams, A., Ireland, J., Luecke, J., McCormick, R.L., and Voorhees, K.J. (2010). Diesel particle filter and fuel effects on heavy-duty diesel engine emissions. Environ. Sci. Technol. 44, 8343-8349. 
Reboul, C., Boissière, J., André, L., Meyer, G., Bideaux, P., Fouret, G., Feillet-Coudray, C., Obert, P., 610 Lacampagne, A., Thireau, J., et al. (2017). Carbon monoxide pollution aggravates ischemic heart 611 failure through oxidative stress pathway. Sci Rep 7, 39715.

612 Robertson, S., Thomson, A.L., Carter, R., Stott, H.R., Shaw, C.A., Hadoke, P.W.F., Newby, D.E., Miller, M.R., and Gray, G.A. (2014). Pulmonary diesel particulate increases susceptibility to myocardial ischemia/reperfusion injury via activation of sensory TRPV1 and $\beta 1$ adrenoreceptors. Part Fibre Toxicol 11, 12 .

Rosca, M.G., Vazquez, E.J., Kerner, J., Parland, W., Chandler, M.P., Stanley, W., Sabbah, H.N., and Hoppel, C.L. (2008). Cardiac mitochondria in heart failure: decrease in respirasomes and oxidative phosphorylation. Cardiovasc. Res. 80, 30-39.

Scheubel, R.J., Tostlebe, M., Simm, A., Rohrbach, S., Prondzinsky, R., Gellerich, F.N., Silber, R.E., and Holtz, J. (2002). Dysfunction of mitochondrial respiratory chain complex I in human failing myocardium is not due to disturbed mitochondrial gene expression. J. Am. Coll. Cardiol. 40, 2174-2181.

Schrepper, A., Schwarzer, M., Schöpe, M., Amorim, P.A., and Doenst, T. (2012). Biphasic response of skeletal muscle mitochondria to chronic cardiac pressure overload - role of respiratory chain complex activity. J. Mol. Cell. Cardiol. 52, 125-135.

Schwarzer, M., Schrepper, A., Amorim, P.A., Osterholt, M., and Doenst, T. (2013). Pressure overload differentially affects respiratory capacity in interfibrillar and subsarcolemmal mitochondria. Am. J. Physiol. Heart Circ. Physiol. 304, H529-537. mitochondrial respiratory chain enzymatic activities on tissues and cultured cells. Nat Protoc 7 , 1235-1246.

Veksler, V.I., Kuznetsov, A.V., Sharov, V.G., Kapelko, V.I., and Saks, V.A. (1987). Mitochondrial respiratory parameters in cardiac tissue: a novel method of assessment by using saponinskinned fibers. Biochim. Biophys. Acta 892, 191-196.

Vergeade, A., Mulder, P., Vendeville-Dehaudt, C., Estour, F., Fortin, D., Ventura-Clapier, R., Thuillez, C., and Monteil, C. (2010). Mitochondrial impairment contributes to cocaine-induced cardiac dysfunction: Prevention by the targeted antioxidant MitoQ. Free Radic. Biol. Med. 49, 748-756. Particulate Air Pollution on the Incidence of Coronary Heart Disease in Shanghai, China. PLoS

642 Yokota, S., Seki, T., Naito, Y., Tachibana, S., Hirabayashi, N., Nakasaka, T., Ohara, N., and 643 Kobayashi, H. (2008). Tracheal instillation of diesel exhaust particles component causes blood 644 and pulmonary neutrophilia and enhances myocardial oxidative stress in mice. J Toxicol Sci 33, 645 609-620. 